\title{
Mediterranean diet impact on changes in abdominal fat and 10-year incidence of abdominal obesity in a Spanish population
}

\author{
Anna N. Funtikova ${ }^{1,2} \dagger$, Alejandra A. Benítez-Arciniega ${ }^{3} \dagger$, Santiago F. Gomez ${ }^{1,4}$, Montserrat Fitó ${ }^{1,5}$, \\ Roberto Elosua ${ }^{6}$ and Helmut Schröder ${ }^{1,2 *}$ \\ ${ }^{1}$ Cardiovascular Risk and Nutrition Research Group (CARIN-ULEC), Research Program in Inflammatory and \\ Cardiovascular Disorders (RICAD), Institut de Recerca Hospital del Mar (IMIM), Biomedical Research Park, \\ c/Doctor Aiguader 88, Barcelona 08003, Spain \\ ${ }^{2}$ CIBER Epidemiology and Public Health (CIBERESP), Instituto de Salud Carlos III, Madrid, Spain, \\ ${ }^{3}$ Faculty of Medicine, Autonomous University of Mexico State, Toluca, Mexico \\ ${ }^{4}$ Fundación THAO, Barcelona, Spain \\ ${ }^{5}$ CIBER Physiopathology of Obesity and Nutrition (CIBEROBN), Instituto de Salud Carlos III, Madrid, Spain \\ ${ }^{6}$ Cardiovascular Epidemiology and Genetics (EGEC-ULEC), Research Program in Inflammatory and Cardiovascular \\ Disorders (RICAD), Institut de Recerca Hospital del Mar (IMIM), Barcelona, Spain
}

(Submitted 9 July 2013 - Final revision received 6 November 2013 - Accepted 12 November 2013 - First published online 2 January 2014)

\section{Abstract}

Abdominal obesity is a strong predictor of metabolic disorders. Prospective data on the association between the Mediterranean diet and surrogate markers of abdominal adiposity are scarce. The present study evaluated the relationship between adherence to the Mediterranean diet and (1) changes in waist circumference (WC) and (2) 10-year incidence of abdominal obesity. We conducted a prospective, populationbased study in 3058 male and female Spaniards aged 25-74 years, followed from 2000 to 2009. Dietary intake and leisure-time physical activity levels were recorded using validated questionnaires. Weight, height and WC were measured. Adherence to the Mediterranean diet, determined using the previously validated REGICOR-Mediterranean diet score (R-MDS), based on the distribution of population food intake and on the dietary recommendations (MDS-rec), was negatively associated with WC gain $(P=0.007$ and 0.024 , respectively) in fully adjusted models. In the multivariate logistic analysis, the odds of abdominal obesity incidence decreased across the tertiles of the R-MDS, but the association was not significant. In conclusion, adherence to the Mediterranean diet was associated with lower abdominal fat gain, but not with 10-year incidence of abdominal obesity.

\section{Key words: Abdominal obesity: Mediterranean diet: Waist circumference}

Abdominal obesity is a strong predictor of metabolic disorders and premature mortality ${ }^{(1,2)}$. Therefore, increasing secular trends of abdominal obesity in recent decades ${ }^{(3)}$ and the recently reported high prevalence of abdominal obesity ${ }^{(3,4)}$ are a matter of health policy concern.

Sedentary lifestyle and dietary patterns promoting energy overconsumption are considered to be the main drivers of the obesity epidemic ${ }^{(5)}$. Epidemiological evidence indicates an inverse association between adherence to the Mediterranean diet and general obesity ${ }^{(6,7)}$. However, little is known about the impact of this dietary pattern, which includes foods characteristic of the Mediterranean olive grove areas, on abdominal fat accumulation ${ }^{(8)}$. An inverse association has been found in some ${ }^{(9)}$ but not in all ${ }^{(10)}$ cross-sectional studies, and long-term prospective data are lacking.

Most studies have measured adherence to the Mediterranean diet by scores based on consumption patterns and modified to reflect the particular study population. This approach makes it difficult to compare results between different populations ${ }^{(11)}$. A Mediterranean diet score has also been created $a$ priori by a few studies ${ }^{(12)}$, using food intake recommendations.

The present study had three objectives: (1) to explore the prospective association of adherence to the Mediterranean diet with waist circumference (WC) changes; (2) to determine the 10-year incidence of abdominal obesity defined by WC cut-offs, according to adherence to the Mediterranean diet in

\footnotetext{
Abbreviations: MDS-rec, Mediterranean diet score based on the dietary recommendations; R-MDS, REGICOR-Mediterranean diet score based on the distribution of population food intake; LTPA, leisure-time physical activity; WC, waist circumference.
}

*Corresponding author: H. Schröder, fax +34933160 796, email hschroeder@imim.es

† A. N. F. and A. A. B.-A. contributed equally to the study, and each can be considered first authors of this article 
a population-based sample in Spain; (3) to assess whether the use of a different strategy to define the Mediterranean diet construct had an impact on the results obtained.

\section{Materials and methods}

\section{Study population}

Data were obtained from a population-based survey conducted in Girona (Spain) in 2000 and 2009. The baseline survey carried out in 2000 examined a randomly selected, population-based sample of 3058 men and women aged 25-74 years (participation rate: $71 \cdot 0 \%$ ). A two-stage sampling method was used in 2000: seventeen towns were randomly selected in the first stage. Half of the towns were urban (>10000 inhabitants) and half were rural (500-10000 inhabitants). The second sampling stage consisted of randomly recruiting the same number of female and male participants, stratifying by 10-year age groups from the closest census.

In 2009, the 2715 non-institutionalised participants still residing in the catchment area were invited for re-examination, and 2181 attended (follow-up participation rate: 80.3\%). Participants following a special diet at re-examination ( $n 207$ (9.8\%)) were excluded. The participants were duly informed, and they signed their consent to participate in the study. The project was approved by the local Ethics Committee (CEIC-PSMAR, Barcelona, Spain).

\section{Anthropometrics}

A calibrated precision scale was used for measuring weight. Readings were rounded up to $200 \mathrm{~g}$. Height was measured in the standing position and rounded up to the nearest $0.5 \mathrm{~cm}$. Weight was divided by height squared $\left(\mathrm{kg} / \mathrm{m}^{2}\right)$ to establish the BMI.

WC was measured midway between the lowest rib and the iliac crest in the horizontal position. Readings were rounded up to the nearest $0.5 \mathrm{~cm}$. Abdominal obesity was defined by sex-specific cut-offs of more than $102 \mathrm{~cm}$ for men and more than $88 \mathrm{~cm}$ for women ${ }^{(13)}$

All the anthropometric parameters were measured both at baseline and at follow-up, using the same protocol.

\section{Dietary intake assessment}

Dietary intake was determined using a validated ${ }^{(14,15)}$ FFQ, administered by a trained interviewer at baseline and at follow-up. In a 166-item food list including alcoholic and non-alcoholic beverages, the participants indicated their usual consumption frequency and chose from ten frequency categories ranging from never or less than once per month to six or more times per $\mathrm{d}$.

Adherence to the Mediterranean diet was determined by the validated REGICOR-Mediterranean diet score (R-MDS) ${ }^{(14)}$. The published Pearson correlation for the energy-adjusted R-MDS $v$. multiple recalls was $0 \cdot 48^{(13)}$. The R-MDS was calculated using two strategies: sex-specific tertile distribution of food intake $^{(14)}$ (R-MDS) and dietary recommendations established in Spain ${ }^{(16,17)}$ (MDS-rec). The lowest tertile was coded as 1 , medium as 2 and the highest as 3 for cereals, fruits, vegetables, legumes, fish, olive oil and nuts. For meat (including red meat, poultry and sausages) and dairy products, the score was inverted, with the highest tertile being coded as 1 and the lowest as 3. Moderate red wine consumption (up to $20 \mathrm{~g} / \mathrm{d}$ ) was coded as 3 , and that more or less than this daily portion was coded as 1 . Using the second strategy, consumption that met the recommendations for cereals, fruits, vegetables, legumes, fish, olive oil, nuts and dairy products was coded as 3, that at least weekly as 2 and that less than weekly levels as 1 for legumes, fish and nuts; consumption at least daily was coded as 2 and that less than daily levels as 1 for cereals, fruits, vegetables, olive oil and dairy products (see Supplementary Appendix 2, available online). For meat (including red meat, poultry and sausages) and dairy products, the score was partially inverted, with consumption more than weekly levels being coded as 1 , that weekly as 2 and that meeting the recommended levels as 3. Red wine consumption was coded as described previously.

\section{Energy misreporting}

Implausible energy reporters were identified by the revised Goldberg method described previously ${ }^{(18)}$. BMR was calculated using the Mifflin equation ${ }^{(19)}$ :

$$
\begin{aligned}
\mathrm{BMR}= & \left(\text { weight }_{\mathrm{kg}} \times 9.99\right)+\left(\text { height }_{\mathrm{cm}} \times 6.25\right)-\left(\text { age }_{\mathrm{y}} \times 4.92\right) \\
& +5(\text { among men }), \\
\mathrm{BMR}= & \left(\text { weight }_{\mathrm{kg}} \times 9.99\right)+\left(\text { height }_{\mathrm{cm}} \times 6.25\right)-\left(\text { age }_{\mathrm{y}} \times 4.92\right) \\
& +161(\text { among women }) .
\end{aligned}
$$

The index of variability $(S)$ in the components of energy balance was determined. Subjects with BMR falling above or below the upper and lower 95\% CI limits of 1.96 standard deviations for plausible energy intake were characterised as implausible energy reporters.

The following formula was used:

$$
\text { Cut-off }=\text { PAL } \times \exp \left[ \pm 1.96 \times \frac{S / 100}{\sqrt{n}}\right],
$$

where

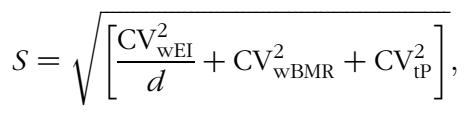

where $d$ is the number of recording days; $\mathrm{CV}_{\mathrm{wEI}}^{2}$ and $\mathrm{CV}_{\text {wBMR }}^{2}$ represent the intra-individual variations in the repeated measures of energy intake and BMR, respectively; and $\mathrm{CV}_{\mathrm{tP}}^{2}$ represents the inter-individual variation in physical activity levels (PAL) ${ }^{(20)}$.

\section{Other variables}

The validated Minnesota leisure-time physical activity (LTPA) questionnaire was administered by a trained interviewer ${ }^{(21,22)}$. LTPA is expressed as metabolic equivalents $\times \mathrm{min} / \mathrm{d}$. 
The measurements of smoking habits and demographic and socio-economic variables were obtained from structured standard questionnaires administered by trained personnel. The participants were dichotomously categorised into nonsmokers (never smokers and ex-smokers with more than 1 year of smoking cessation) and current smokers. Maximum educational level attained was determined and dichotomously recorded for analysis as primary school $v$. secondary school or university.

All dietary and energy intake and expenditure measurements were performed both at baseline and at follow-up, using the same protocol.

\section{Statistical analysis}

General linear modelling procedures were used to compare the baseline characteristics of the participants by tertiles of the R-MDS and by categories of the MDS-rec. ANOVA test and polynomial contrasts were used to determine overall $P$ and $P$ for linear trend, respectively, for normally distributed continuous variables. The Kruskal-Wallis test was used to determine overall $P$ for non-normal distribution. Overall $P$ and $P$ for linear trend for categorical variables were obtained using the Pearson $\chi^{2}$ and Mantel-Haenszel linear-by-linear association $\chi^{2}$ tests, respectively.

Linear regression models were fitted to analyse the association between changes in WC and adherence to the Mediterranean diet at baseline. Changes in WC (in $\mathrm{cm}$; continuous variable) were included as the dependent variable and scores as explanatory variables. For analyses, three models were fitted. The first model included sex (men/women; dichotomous), age (years; continuous) and baseline WC. The second model included smoking (yes/no; dichotomous), energy intake (kcal; continuous), alcohol consumption (g, continuous), educational level (more than primary school yes/no; dichotomous), LTPA (metabolic equivalents $\times \mathrm{min} / \mathrm{d}$; continuous), and energy under- and over-reporting (both yes/no; dichotomous). The third model included changes in BMI (BMI at follow-up - BMI at baseline; continuous) to determine whether changes in WC are independent of changes in BMI. This model was adjusted by sex (men/ women; dichotomous), age (years; continuous), smoking (yes/no; dichotomous), energy intake (kcal; continuous), alcohol consumption (g; continuous), educational level (more than primary school yes/no; dichotomous), LTPA (metabolic equivalents $\times \mathrm{min} / \mathrm{d}$; continuous), and energy

Table 1. General characteristics of the sample at baseline according to tertiles of the REGICOR-Mediterranean diet score (R-MDS) ${ }^{\star}$

\begin{tabular}{|c|c|c|c|c|c|c|c|c|}
\hline \multirow[b]{3}{*}{ Variables } & \multicolumn{6}{|c|}{ R-MDS tertiles } & \multirow[b]{3}{*}{$P \dagger$} & \multirow[b]{3}{*}{$P \neq$} \\
\hline & \multicolumn{2}{|c|}{ 1st $(n 650)$} & \multicolumn{2}{|c|}{ 2nd ( $n$ 618) } & \multicolumn{2}{|c|}{ 3rd $(n 611)$} & & \\
\hline & Proportions & $95 \% \mathrm{Cl}$ & Proportions & $95 \% \mathrm{Cl}$ & Proportions & $95 \% \mathrm{Cl}$ & & \\
\hline Women (\%) & $51 \cdot 4$ & $47 \cdot 5,55 \cdot 2$ & $53 \cdot 1$ & $49 \cdot 1,57 \cdot 0$ & $50 \cdot 4$ & $46 \cdot 4,54 \cdot 4$ & 0.639 & 0.741 \\
\hline Age (years) & & & & & & & $<0.001$ & $<0.001$ \\
\hline Mean & \multicolumn{2}{|c|}{47.4} & \multicolumn{2}{|c|}{49.9} & 49.8 & & & \\
\hline $95 \% \mathrm{Cl}$ & \multicolumn{2}{|c|}{$46 \cdot 4,48 \cdot 4$} & \multicolumn{2}{|c|}{$48 \cdot 9,50 \cdot 9$} & \multicolumn{2}{|c|}{$48 \cdot 8,50 \cdot 8$} & & \\
\hline Education $(\%) \S$ & 37.5 & $33 \cdot 8,41 \cdot 3$ & 34.8 & $31 \cdot 0,38 \cdot 6$ & $38 \cdot 1$ & $34 \cdot 3,42 \cdot 0$ & 0.429 & 0.887 \\
\hline Smokers (\%)\| & $30 \cdot 3$ & $26 \cdot 9,33 \cdot 7$ & $24 \cdot 1$ & $20 \cdot 7,27 \cdot 6$ & 23.4 & $19 \cdot 9,26 \cdot 9$ & 0.008 & 0.005 \\
\hline LTPA $($ MET $\times$ min/d) & & & & & & & $<0.001$ & - \\
\hline Median & \multicolumn{2}{|c|}{167} & \multicolumn{2}{|c|}{190} & \multicolumn{2}{|c|}{240} & & \\
\hline 25th-75th percentiles & \multicolumn{2}{|c|}{$87-321$} & \multirow{2}{*}{\multicolumn{2}{|c|}{$89-338$}} & \multicolumn{2}{|c|}{$130-396$} & & \\
\hline WC $(\mathrm{cm})$ & & & & & & & 0.387 & 0.387 \\
\hline Mean & \multicolumn{2}{|c|}{89.6} & \multicolumn{2}{|c|}{89.5} & \multicolumn{2}{|c|}{89.0} & & \\
\hline $95 \% \mathrm{Cl}$ & \multicolumn{2}{|c|}{$88 \cdot 6,90 \cdot 7$} & \multicolumn{2}{|c|}{$88 \cdot 4,90 \cdot 6$} & \multicolumn{2}{|c|}{$87 \cdot 9,90 \cdot 1$} & & \\
\hline WC at follow-up (cm) & & & & & & & 0.030 & 0.030 \\
\hline Mean & \multicolumn{2}{|c|}{$94 \cdot 2$} & \multicolumn{2}{|c|}{$93 \cdot 4$} & \multicolumn{2}{|c|}{$92 \cdot 6$} & & \\
\hline $95 \% \mathrm{Cl}$ & \multicolumn{2}{|c|}{$93 \cdot 2,95 \cdot 2$} & \multicolumn{2}{|c|}{$92 \cdot 4,94 \cdot 4$} & \multicolumn{2}{|c|}{$91 \cdot 6,93.6$} & & \\
\hline Abdominal obesity (\%) & 30.6 & $27 \cdot 1,34 \cdot 1$ & $32 \cdot 2$ & $28 \cdot 6,35 \cdot 8$ & 24.9 & $21 \cdot 3,28 \cdot 5$ & 0.028 & 0.028 \\
\hline Abdominal obesity at follow-up (\%) & $44 \cdot 2$ & $40 \cdot 4,47.9$ & $42 \cdot 4$ & $38 \cdot 5,46 \cdot 3$ & $35 \cdot 7$ & $31 \cdot 8,39 \cdot 6$ & 0.002 & 0.002 \\
\hline Alcohol consumption (g/d) & & & & & & & 0.001 & - \\
\hline Mean & & & & & & & & \\
\hline $95 \% \mathrm{Cl}$ & & & & & $2 \cdot 1$, & & & \\
\hline Energy (MJ) & & & & & & & $<0.001$ & $<0.001$ \\
\hline Mean & & & & & 13 & & & \\
\hline $95 \% \mathrm{Cl}$ & & & $10 \cdot 8$ & 1.5 & $12 \cdot 7$ & $3 \cdot 3$ & & \\
\hline Energy under-reporters (\%) & $35 \cdot 2$ & $32 \cdot 1,38 \cdot 4$ & $20 \cdot 1$ & $16 \cdot 8,23 \cdot 3$ & $12 \cdot 8$ & $9 \cdot 5,16 \cdot 0$ & $<0.001$ & $<0.001$ \\
\hline Energy over-reporters (\%) & 5.4 & $2 \cdot 9,7.8$ & $11 \cdot 7$ & $9 \cdot 1,14 \cdot 2$ & $19 \cdot 0$ & $16 \cdot 5,21 \cdot 5$ & $<0.001$ & $<0.001$ \\
\hline
\end{tabular}

LTPA, leisure-time physical activity; MET, metabolic equivalents; WC, waist circumference.

${ }^{*}$ Age, WC, WC at follow-up, alcohol consumption and energy are continuous variables; women, education, smokers, abdominal obesity, abdominal obesity at follow-up, energy under-reporters and energy over-reporters are categorical variables.

† $P$ values were obtained using the ANOVA, Kruskal-Wallis and Pearson $\chi^{2}$ tests for normal continuous, non-normal continuous and categorical variables, respectively.

$\ddagger P$ values for linear trend were obtained using polynomial contrast for normal continuous variables and using the Mantel-Haenszel linear-by-linear association $\chi^{2}$ test for categorical variables.

$\S$ More than secondary school education.

\| Active smokers or ex-smokers for less than 1 year.

If $W C>102 \mathrm{~cm}$ in men and $>88 \mathrm{~cm}$ in women. 
under- and over-reporting (both yes/no; dichotomous). Participants with baseline abdominal obesity were excluded from analysis in multiple logistic regression models assessing the association between R-MDS and MDS-rec adherence at baseline and 10-year incidence of abdominal obesity. Baseline R-MDS and MDS-rec values are represented by tertiles and categories, respectively.

To explore effect modification according to sex, R-MDS and MDS-rec, we modelled interaction terms for sex/R-MDS and sex/MDS-rec. Differences were considered significant if $P<0 \cdot 05$. Statistical analysis was carried out using SPSS version 18.0. (SPSS, Inc.).

\section{Results}

The prevalence of abdominal obesity at baseline was $29 \cdot 3 \%$ (men: $23.8 \%$; women: $34.4 \%$ ) and at follow-up was $40 \cdot 8 \%$ (men: 30.5\%; women: 50.5\%). Of the 1329 participants without abdominal obesity at baseline, 291 (21.9\%) had abdominal obesity at follow-up (men: 14.9\%; women: $29 \cdot 6 \%$ ).

Participants with high adherence to the Mediterranean diet were less prone to smoke and had a lower prevalence of abdominal obesity than those with low dietary adherence
(Tables 1 and 2). Age, LTPA, alcohol consumption and energy intake increased across the R-MDS tertiles and MDS-rec categories. The proportion of energy under-reporters decreased with greater adherence to the Mediterranean diet; with lesser adherence, energy over-reporting increased.

Linear regression analysis revealed a significant negative association of WC gain with the R-MDS and MDS-rec ( $P=0.007$ and 0.024 , respectively) in the fully adjusted models (Table 3). A 10-point increase in the R-MDS and MDS-rec was associated with a decrease of 1.7 and $1.5 \mathrm{~cm}$ in WC, respectively. The association of changes in WC with adherence to the Mediterranean diet was independent of BMI changes.

In the multivariate logistic analysis, high adherence to the Mediterranean diet (top tertile) was associated with a reduced 10 -year incidence of abdominal obesity, but this association did not reach significance (Table 4). The magnitude of risk reduction was similar for both scores.

\section{Discussion}

In the present prospective study, it was found that high adherence to the Mediterranean diet was associated with a

Table 2. General characteristics of the sample at baseline according to categories of the Mediterranean diet score based on dietary recommendations (MDS-rec)*

\begin{tabular}{|c|c|c|c|c|c|c|c|c|}
\hline \multirow[b]{3}{*}{ Variables } & \multicolumn{6}{|c|}{ MDS-rec categories } & \multirow[b]{3}{*}{$P \dagger$} & \multirow[b]{3}{*}{$P \neq$} \\
\hline & \multicolumn{2}{|c|}{ 1st $(n 693)$} & \multicolumn{2}{|c|}{ 2nd ( $n$ 507) } & \multicolumn{2}{|c|}{$3 r d(n 679)$} & & \\
\hline & Proportions & $95 \% \mathrm{Cl}$ & Proportions & $95 \% \mathrm{Cl}$ & Proportions & $95 \% \mathrm{Cl}$ & & \\
\hline Women (\%) & $50 \cdot 1$ & $46 \cdot 3,53 \cdot 8$ & $50 \cdot 1$ & $45 \cdot 7,54 \cdot 5$ & $54 \cdot 3$ & $50 \cdot 6,58 \cdot 1$ & 0.207 & 0.114 \\
\hline Age (years) & & & & & & & 0.021 & 0.089 \\
\hline Mean & \multicolumn{2}{|c|}{$48 \cdot 0$} & \multicolumn{2}{|c|}{$50 \cdot 1$} & \multicolumn{2}{|c|}{$49 \cdot 2$} & & \\
\hline $95 \% \mathrm{Cl}$ & \multicolumn{2}{|c|}{$47 \cdot 1,49 \cdot 0$} & \multicolumn{2}{|c|}{$49 \cdot 0,51 \cdot 2$} & \multicolumn{2}{|c|}{$48 \cdot 2,50 \cdot 2$} & & \\
\hline Education $(\%) \S$ & 36.9 & $33 \cdot 3,40 \cdot 5$ & 34.5 & $30 \cdot 3,38 \cdot 7$ & 38.4 & $34 \cdot 8,42 \cdot 1$ & 0.382 & 0.570 \\
\hline Smokers (\%)\| & $31 \cdot 2$ & $27 \cdot 9,34 \cdot 4$ & $26 \cdot 8$ & $23 \cdot 0,30 \cdot 6$ & $20 \cdot 2$ & $16 \cdot 9,23 \cdot 5$ & $<0.001$ & $<0.001$ \\
\hline LTPA $(\mathrm{MET} \times \mathrm{min} / \mathrm{d})$ & & & & & & & $<0.001$ & - \\
\hline Median & \multicolumn{2}{|c|}{171} & \multicolumn{2}{|c|}{190} & \multicolumn{2}{|c|}{232} & & \\
\hline 25th-75th percentiles & \multicolumn{2}{|c|}{$84-322$} & \multicolumn{2}{|c|}{$105-334$} & \multicolumn{2}{|c|}{$117-387$} & & \\
\hline $\mathrm{WC}(\mathrm{cm})$ & \multirow{2}{*}{\multicolumn{2}{|c|}{89.4}} & & & & & 0.324 & 0.112 \\
\hline Mean & & & \multicolumn{2}{|c|}{$90 \cdot 3$} & \multicolumn{2}{|c|}{88.6} & & \\
\hline $95 \% \mathrm{Cl}$ & \multicolumn{2}{|c|}{$88 \cdot 4,90 \cdot 4$} & \multicolumn{2}{|c|}{$89 \cdot 1,91 \cdot 5$} & \multicolumn{2}{|c|}{$87 \cdot 6,89 \cdot 7$} & & \\
\hline WC at follow-up $(\mathrm{cm})$ & & \multirow{2}{*}{\multicolumn{2}{|c|}{94.6}} & & & 0.026 & 0.005 \\
\hline Mean & \multirow{2}{*}{\multicolumn{2}{|c|}{$\begin{array}{c}93 \cdot 8 \\
92 \cdot 8,94 \cdot 7\end{array}$}} & & & \multicolumn{2}{|c|}{$92 \cdot 2$} & & \\
\hline $95 \% \mathrm{Cl}$ & & & \multicolumn{2}{|c|}{$93 \cdot 5,95 \cdot 7$} & \multicolumn{2}{|c|}{$91 \cdot 3,93 \cdot 2$} & & \\
\hline Abdominal obesity (\%) & $29 \cdot 7$ & $26 \cdot 3,33 \cdot 1$ & $32 \cdot 7$ & $28 \cdot 8,36 \cdot 7$ & $26 \cdot 2$ & $22 \cdot 8,29 \cdot 6$ & 0.156 & 0.048 \\
\hline Abdominal obesity at follow-up (\%) & $42 \cdot 0$ & $38 \cdot 3,45 \cdot 6$ & $44 \cdot 6$ & $40 \cdot 3,48 \cdot 9$ & $36 \cdot 8$ & $33 \cdot 1,40 \cdot 5$ & 0.053 & 0.020 \\
\hline Alcohol consumption $(\mathrm{g} / \mathrm{d})$ & & & & & & & 0.001 & - \\
\hline Mean & 2 & & & & & & & \\
\hline $95 \% \mathrm{Cl}$ & 0,2 & & & & 1.9 & & & \\
\hline Energy (MJ) & & & & & & & $<0.001$ & $<0.001$ \\
\hline Mean & 9 & & & & & & & \\
\hline $95 \% \mathrm{Cl}$ & $9 \cdot 1$ & & $10 \cdot \varepsilon$ & 1.5 & $12 \cdot 8$ & 13.4 & & \\
\hline Energy under-reporters (\%) & 37.5 & $34.5,40.5$ & $20 \cdot 5$ & $17 \cdot 0,24 \cdot 0$ & $9 \cdot 9$ & $6 \cdot 8,12 \cdot 9$ & $<0.001$ & $<0.001$ \\
\hline Energy over-reporters $(\%)$ & $5 \cdot 2$ & $2 \cdot 8,7 \cdot 6$ & 10.5 & $7 \cdot 7,13 \cdot 2$ & 19.7 & $17 \cdot 3,22 \cdot 1$ & $<0.001$ & $<0.001$ \\
\hline
\end{tabular}

LTPA, leisure-time physical activity; MET, metabolic equivalents; WC, waist circumference.

${ }^{*}$ Age, WC, WC at follow-up, alcohol consumption and energy are continuous variables; women, education, smokers, abdominal obesity, abdominal obesity at follow-up, energy under-reporters and energy over-reporters are categorical variables.

$\dagger P$ values were obtained using the ANOVA, Kruskal-Wallis and Pearson $\chi^{2}$ tests for normal continuous, non-normal continuous and categorical variables, respectively.

$\ddagger P$ values for linear trend were obtained using polynomial contrast for normal continuous variables and using the Mantel-Haenszel linear-by-linear association $\chi^{2}$ test for categorical variables.

$\S$ More than secondary school education.

$\|$ Active smokers or ex-smokers for less than 1 year.

If $W C>102 \mathrm{~cm}$ in men and $>88 \mathrm{~cm}$ in women. 
Table 3. Multiple linear regression analysis of the association between 10-point increase in the REGICOR-Mediterranean diet score (R-MDS) and the Mediterranean diet score based on dietary recommendations (MDS-rec) and 10-year changes in waist circumference (WC) (in $\mathrm{cm}$ )

( $\beta$-Coefficients and $95 \%$ confidence intervals)

\begin{tabular}{|c|c|c|c|}
\hline & $\beta$ & $95 \% \mathrm{Cl}$ & $P$ \\
\hline \multicolumn{4}{|l|}{ R-MDS } \\
\hline Model $1^{*}$ & -1.50 & $-2.74,-0.25$ & 0.019 \\
\hline Model $2 \dagger$ & -1.55 & $-2 \cdot 88,-0.21$ & 0.033 \\
\hline Model 3ł & -1.65 & $-2.84,-0.45$ & 0.007 \\
\hline \multicolumn{4}{|l|}{ MDS-rec } \\
\hline Model $1^{*}$ & -1.53 & $-2 \cdot 87,0.19$ & 0.259 \\
\hline Model $2 \dagger$ & -1.64 & $-3.16,-0.13$ & 0.033 \\
\hline Model 3‡ & -1.49 & $-2.85,-0.13$ & 0.024 \\
\hline
\end{tabular}

* Model 1 includes sex (men/women; dichotomous), age (years; continuous) and baseline WC (cm; continuous).

† Model 2 additionally includes smoking (yes/no; dichotomous), energy intake (kcal; continuous), alcohol consumption (g; continuous), educational level (more than primary school yes/no; dichotomous), leisure-time physical activity (metabolic equivalents $\times \mathrm{min} / \mathrm{d}$; continuous), and energy under- and over-reporting (both yes/no; dichotomous).

¥ Model 3 includes variables of model 2 and changes in BMI (BMI at follow-up - BMI at baseline; continuous)

lower gain in abdominal adiposity but not with the incidence of abdominal obesity within 10 years. In recent decades, increased WC has been reported for several populations ${ }^{(3)}$. In the present study, general obesity was about $7 \%$ less prevalent than abdominal obesity at baseline. Most importantly, 10-year incidence of abdominal obesity was $14 \cdot 8 \%$ higher than that of general obesity. This is a cause of concern due to the direct association between WC and cardiometabolic risk ${ }^{(2)}$.

A recently published meta-analysis ${ }^{(23)}$ of the impact of adherence to the Mediterranean diet on the metabolic syndrome and its components has revealed a positive effect on WC changes in eleven randomised controlled trials. It is worth noting that this was mainly due to one trial that demonstrated a mean WC difference of $-4.20 \mathrm{~cm}(-7.99$ to $-0 \cdot 41)$ between the Mediterranean and control diets ${ }^{(24)}$.

Among the prospective observational studies, only three surveys ${ }^{(11,25,26)}$ evaluated the association between the Mediterranean diet and WC changes. In the SUN prospective cohort (Seguimiento Universidad de Navarra; University of Navarra follow-up) the difference in WC between the highest and lowest tertiles of the Mediterranean diet score in sexand age-adjusted models was almost $0^{(26)}$. Participants in the Framingham Heart Study Offspring Cohort who closely adhered to the Mediterranean diet had a significantly lower WC than their low-adhering peers ${ }^{(25)}$. Additionally, the SUpplementation en VItamines et Minéraux AntioXydants (SU.VI.MAX) study reported a negative association between $\mathrm{WC}$ gain and two of the three Mediterranean diet score indices calculated ${ }^{(11)}$.

Our data confirm the findings of previous studies showing a protective association of the Mediterranean diet with abdominal adiposity gain. In the present study, a 10-point increase in dietary scores was associated with a $1.7 \mathrm{~cm}$ decrease in WC.

Cross-sectional studies ${ }^{(23,27)}$ have reported mixed results, which might be due to differences in the surrogate markers of abdominal obesity used (WC, waist:hip ratio and waist: height ratio). In the present study, cross-sectional analysis revealed that the risk of abdominal obesity decreased with high adherence to the Mediterranean diet.

Some ${ }^{(6,28,29)}$ but not all ${ }^{(30,31)}$ prospective studies provide evidence for the protective effects of the Mediterranean diet on the incidence of general obesity. To our knowledge, no previous study has addressed the association between the Mediterranean diet and incidence of abdominal obesity. We defined abdominal obesity by WC, a surrogate marker of abdominal adiposity. We observed an inverse, but non-significant, association between adherence to the Mediterranean diet and incidence of abdominal obesity. One explanation for these results is the moderate magnitude of the association of WC change with the R-MDS. On the other hand, a substantial increase in the prevalence of abdominal obesity at follow-up and low mean change in the R-MDS ( -0.5 points) during 10 years cancelled the effect of the Mediterranean diet on the incidence of abdominal obesity. This outcome is of interest at the public health level because it is also important to know which diets do not promote abdominal obesity.

Higher adherence to the Mediterranean diet was associated with high consumption of MUFA and PUFA, high dietary fibre intake and low energy density (data not shown). High consumption of fibre might partially explain the impact of the Mediterranean diet on abdominal adiposity through various protective mechanisms, with the increase of satiety and decrease of energy consumption ${ }^{(32,33)}$, modification of hepatic cholesterol metabolism ${ }^{(30)}$, and increased production of SCFA being among them ${ }^{(34)}$. Du et al. $^{(35)}$ found that a $1 \mathrm{kcal} / \mathrm{g}$ $(4 \mathrm{~kJ} / \mathrm{g})$ increase in energy density was associated with a $0.09 \mathrm{~cm}$ gain in WC per year. It has been hypothesised that energy density could be a mediator between depressive symptoms and abdominal adiposity gain ${ }^{(36)}$. However, the

Table 4. OR of 10-year incidence of abdominal obesity according to tertile distribution of the REGICOR-Mediterranean diet score (R-MDS) and categories of the Mediterranean diet score based on dietary recommendations (MDS-rec)*

(Odds ratios and $95 \%$ confidence intervals)

\begin{tabular}{|c|c|c|c|c|c|c|}
\hline & \multicolumn{5}{|c|}{ R-MDS tertiles and MDS-rec categories } & \multirow[b]{3}{*}{$P$} \\
\hline & \multirow[b]{2}{*}{$1^{\text {st }}$} & \multicolumn{2}{|r|}{ 2nd } & \multicolumn{2}{|r|}{ 3rd } & \\
\hline & & OR & $95 \% \mathrm{Cl}$ & OR & $95 \% \mathrm{Cl}$ & \\
\hline \multicolumn{7}{|l|}{ R-MDS } \\
\hline Model 1† & Ref. & 0.70 & $0.50,0.97$ & 0.75 & $0.55,1.04$ & 0.085 \\
\hline Model $2 \ddagger$ & Ref. & 0.73 & $0.52,1.04$ & 0.79 & $0.55,1.12$ & 0.185 \\
\hline \multicolumn{7}{|l|}{ MDS-rec } \\
\hline Model $1 \dagger$ & Ref. & 0.99 & $0.70,1.34$ & 0.81 & $0.59,1 \cdot 11$ & 0.186 \\
\hline Model $2 \ddagger$ & Ref. & 1.08 & $0.76,1.55$ & 0.90 & $0.64,1.29$ & 0.571 \\
\hline
\end{tabular}

Ref., reference.

*Total number of participants $=1329$; distribution of the participants according to tertiles of the R-MDS: 1 st tertile $=451$; 2 nd tertile $=419$; 3rd tertile $=459$; distribution of the participants according to categories of the MDS-rec: 1st category $=487 ;$ 2nd category $=341 ;$ 3rd category $=501$.

† Model 1 includes sex (men/women; dichotomous), age (years; continuous) and baseline waist circumference (cm; continuous).

‡ Model 2 additionally includes smoking (yes/no; dichotomous), energy intake (kcal; continuous), alcohol consumption (g; continuous), educational level (more than primary school yes/no; dichotomous), leisure-time physical activity (metabolic equivalents $\times \mathrm{min} / \mathrm{d}$; continuous), and energy under- and over-reporting (both yes/no; dichotomous). 
mechanisms linking WC changes and energy density are not understood. Fatty acids can activate the PPAR $\gamma$ gene ${ }^{(37)}$, which is involved in lipid metabolism and particularly in the process of adipocyte differentiation. It has been demonstrated that the WC gain caused by the 12Ala allele carriers of the PPAR $\gamma$ gene was depressed by adherence to the Mediterranean $\operatorname{diet}^{(38)}$.

Assessing the adherence to the Mediterranean diet by a score based on population-based food consumption distribution is specific to a particular population, making it difficult to compare results between studies. This construct has mainly been used by studies reporting on the association between adherence to the Mediterranean diet and incidence of obesity and/or changes in anthropometric markers of adiposity ${ }^{(11,27)}$. To overcome the limitation on comparability of results, we calculated two scores, one based on food distribution and the other based on food intake recommendations. We observed no meaningful impact of the type of construct used on the association between the score obtained and WC or incidence of abdominal obesity.

The strengths of the present study are the population-based design, the high participation rate during follow-up, and the measurement of anthropometric variables at baseline and at 10-year follow-up. Furthermore, dietary intake and LTPA data were recorded using validated questionnaires and all the analyses were controlled for misreporting. A benefit of using the MDS-rec is that it allows generalisability to other populations. However, all the dietary instruments measuring past food intake are vulnerable to random and systematic measurement errors.

In conclusion, adherence to the Mediterranean diet was negatively associated with changes in abdominal adiposity measured by WC. Participants with high adherence to the Mediterranean diet were less likely to develop abdominal obesity according to R-MDS tertile distribution and MDS-rec categories. However, the association was not significant. The type of R-MDS calculation (population distribution $v$. dietary recommendations) did not have a strong impact on these associations. Evidence of the present study underlines the usefulness of the Mediterranean diet for the prevention of abdominal weight gain.

\section{Supplementary material}

To view supplementary material for this article, please visit http://dx.doi.org/10.1017/S0007114513003966

\section{Acknowledgements}

The authors thank Elaine Lilly, PhD (Writers First Aid), for revising the manuscript for English language.

The present study was supported by a grant (2FD097-0297CO2-01) from Fondo Europeo de Desarrollo Regional (FEDER); by portions of grants from Spain's Ministerio de Sanidad y Consumo, Instituto de Salud Carlos III FEDER (PI11/01900), Red HERACLES (RD06/0009), by scholarship for $\mathrm{PhD}$ formation from the National Program of University Professors Formation (FPU), from the Ministry of Education of Spain (AP2010-3198), and by a joint Miguel Servet contract (CP03/00115) from the Instituto de Salud Carlos III and the Health Department of the Catalan Government
(Generalitat de Catalunya). The CIBERESP is an initiative of the Instituto de Salud Carlos III, Madrid, Spain.

The authors' contributions are as follows: A. N. F. and A. A. B.-A. prepared the manuscript, with significant input and feedback from all the co-authors; S. F. G., M. F. and R. E. participated in the design and execution of the study and contributed to the critical revision of the manuscript for important intellectual content; H. S. conducted the analyses, with results being interpreted by all the co-authors. All the authors approved the final version of the manuscript.

None of the authors has any conflicts of interest to declare.

\section{References}

1. Cameron AJ, Dunstan DW, Owen N, et al. (2009) Health and mortality consequences of abdominal obesity: evidence from the AusDiab study. Med J Aust 191, 202-208.

2. Fox KA, Despres JP, Richard AJ, et al. (2009) Does abdominal obesity have a similar impact on cardiovascular disease and diabetes? A study of 91,246 ambulant patients in 27 European countries. Eur Heart J 30, 3055-3063.

3. Ford ES, Mokdad AH \& Giles WH (2003) Trends in waist circumference among U.S. adults. Obes Res 11, 1223-1231.

4. Gutierrez-Fisac JL, Guallar-Castillon P, Leon-Munoz LM, et al. (2012) Prevalence of general and abdominal obesity in the adult population of Spain, 2008-2010: the ENRICA study. Obes Rev 13, 388-392.

5. Apovian CM (2010) The causes, prevalence, and treatment of obesity revisited in 2009: what have we learned so far? Am J Clin Nutr 91, 277S-279S.

6. Romaguera D, Norat T, Vergnaud AC, et al. (2010) Mediterranean dietary patterns and prospective weight change in participants of the EPIC-PANACEA project. Am J Clin Nutr 92, 912-921.

7. Buckland G, Bach A \& Serra-Majem L (2008) Obesity and the Mediterranean diet: a systematic review of observational and intervention studies. Obes Rev 9, 582-593.

8. Rallidis LS, Lekakis J, Kolomvotsou A, et al. (2009) Close adherence to a Mediterranean diet improves endothelial function in subjects with abdominal obesity. Am J Clin Nutr 90, 263-268.

9. Romaguera D, Norat T, Mouw T, et al. (2009) Adherence to the Mediterranean diet is associated with lower abdominal adiposity in European men and women. J Nutr 139, 1728-1737.

10. Asghari G, Mirmiran P, Rashidkhani B, et al. (2012) The association between diet quality indices and obesity: Tehran Lipid and Glucose Study. Arch Iran Med 15, 599-605.

11. Kesse-Guyot E, Ahluwalia N, Lassale C, et al. (2012) Adherence to Mediterranean diet reduces the risk of metabolic syndrome: a 6-year prospective study. Nutr Metab Cardiovasc Dis 3, 677-683.

12. Rumawas ME, Dwyer JT, McKeown NM, et al. (2009) The development of the Mediterranean-style dietary pattern score and its application to the American diet in the Framingham Offspring Cohort. J Nutr 139, 1150-1156.

13. Lean ME, Han TS \& Morrison CE (1995) Waist circumference as a measure for indicating need for weight management. BMJ 311, 158-161.

14. Benitez-Arciniega AA, Mendez MA, Baena-DiezJM, et al. (2011) Concurrent and construct validity of Mediterranean diet scores as assessed by an FFQ. Public Health Nutr 14, 2015-2021.

15. Schroder H, Covas MI, Marrugat J, et al. (2001) Use of a three-day estimated food record, a 72-hour recall and a food-frequency questionnaire for dietary assessment in a Mediterranean Spanish population. Clin Nutr 20, 429-437. 
16. Agencia Española de Seguridad Alimentaria y Nutrición Ministerio de Sanidad y Consumo (2008) Come sano y muévete. 12 decisiones saludables (Eat healthy and move. 12 healthy decisions). AESAN.

17. Sociedad Española de Dietética y Ciencias de Alimentación (2009) Recomendaciones SEDCA 2009 para mantener una alimentación saludable (SEDCA 2009 Recommendations to maintain a healthy diet). SEDCA.

18. Mendez MA, Popkin BM, Buckland G, et al. (2011) Alternative methods of accounting for underreporting and overreporting when measuring dietary intake-obesity relations. Am J Epidemiol 173, 448-458.

19. Mifflin MD, St Jeor ST, Hill LA, et al. (1990) A new predictive equation for resting energy expenditure in healthy individuals. Am J Clin Nutr 51, 241-247.

20. Black AE (2000) Critical evaluation of energy intake using the Goldberg cut-off for energy intake: basal metabolic rate. A practical guide to its calculation, use and limitations. Int J Obes Relat Metab Disord 24, 1119-1130.

21. Elosua R, Marrugat J, Molina L, et al. (1994) Validation of the Minnesota Leisure Time Physical Activity Questionnaire in Spanish men. The MARATHOM Investigators. Am J Epidemiol 139, 1197-1209.

22. Elosua R, Garcia M, Aguilar A, et al. (2000) Validation of the Minnesota Leisure Time Physical Activity Questionnaire in Spanish Women. Investigators of the MARATDON Group. Med Sci Sports Exerc 32, 1431-1437.

23. Kastorini CM, Milionis HJ, Esposito K, et al. (2011) The effect of Mediterranean diet on metabolic syndrome and its components: a meta-analysis of 50 studies and 534,906 individuals. J Am Coll Cardiol 57, 1299-1313.

24. McManus K, Antinoro L \& Sacks F (2001) A randomized controlled trial of a moderate-fat, low-energy diet compared with a low fat, low-energy diet for weight loss in overweight adults. Int J Obes Relat Metab Disord 25, 1503-1511.

25. Rumawas ME, Meigs JB, Dwyer JT, et al. (2009) Mediterranean-style dietary pattern, reduced risk of metabolic syndrome traits, and incidence in the Framingham Offspring Cohort. Am J Clin Nutr 90, 1608-1614.

26. Tortosa A, Bes-Rastrollo M, Sanchez-Villegas A, et al. (2007) Mediterranean diet inversely associated with the incidence of metabolic syndrome: the SUN prospective cohort. Diabetes Care 30, 2957-2959.
27. Martinez-Gonzalez MA, Garcia-Arellano A, Toledo E, et al. (2012) A 14-item Mediterranean diet assessment tool and obesity indexes among high-risk subjects: the PREDIMED trial. PLOS ONE 7, e43134.

28. Mendez MA, Popkin BM, Jakszyn P, et al. (2006) Adherence to a Mediterranean diet is associated with reduced 3-year incidence of obesity. J Nutr 136, 2934-2938.

29. May AM, Romaguera D, Travier N, et al. (2012) Combined impact of lifestyle factors on prospective change in body weight and waist circumference in participants of the EPIC-PANACEA study. PLOS ONE 7, e50712.

30. Beunza JJ, Toledo E, Hu FB, et al. (2010) Adherence to the Mediterranean diet, long-term weight change, and incident overweight or obesity: the Seguimiento Universidad de Navarra (SUN) cohort. Am J Clin Nutr 92, 1484-1493.

31. Yannakoulia M, Panagiotakos D, Pitsavos C, et al. (2009) Five-year incidence of obesity and its determinants: the ATTICA study. Public Health Nutr 12, 36-43.

32. Galisteo M, Duarte J \& Zarzuelo A (2008) Effects of dietary fibers on disturbances clustered in the metabolic syndrome. J Nutr Biochem 19, 71-84.

33. Schroder H (2007) Protective mechanisms of the Mediterranean diet in obesity and type 2 diabetes. $J$ Nutr Biochem 18, 149-160.

34. Hu GX, Chen GR, Xu H, et al. (2010) Activation of the AMP activated protein kinase by short-chain fatty acids is the main mechanism underlying the beneficial effect of a high fiber diet on the metabolic syndrome. Med Hypotheses 74, 123-126.

35. Du H, van der AD, Ginder V, et al. (2009) Dietary energy density in relation to subsequent changes of weight and waist circumference in European men and women. PLOS ONE 4, e5339.

36. Grossniklaus DA, Dunbar SB, Gary R, et al. (2012) Dietary energy density: a mediator of depressive symptoms and abdominal obesity or independent predictor of abdominal obesity? Eur J Cardiovasc Nurs 11, 423-431.

37. Xu HE, Lambert MH, Montana VG, et al. (1999) Molecular recognition of fatty acids by peroxisome proliferatoractivated receptors. Mol Cell 3, 397-403.

38. Razquin C, Alfredo MJ, Martinez-Gonzalez MA, et al. (2009) The Mediterranean diet protects against waist circumference enlargement in 12Ala carriers for the PPAR $\gamma$ gene: 2 years' follow-up of 774 subjects at high cardiovascular risk. $\mathrm{BrJ}$ Nutr 102, 672-679. 